Under consideration for publication in Math. Struct. in Comp. Science

\title{
Well-Founded Coalgebras, Revisited
}

\author{
Jean-Baptiste Jeannin ${ }^{1}$, Dexter Kozen ${ }^{1}$, Alexandra Silva ${ }^{2}$ \\ ${ }^{1}$ Cornell University, Ithaca, $N Y$ \\ ${ }^{2}$ Radboud University Nijmegen, The Netherlands
}

Received 24 May 2013

\begin{abstract}
Theoretical models of recursion schemes have been well studied under the names well-founded coalgebras, recursive coalgebras, corecursive algebras, and Elgot algebras. Much of this work focuses on conditions ensuring unique or canonical solutions, e.g. when the coalgebra is well-founded.

If the coalgebra is not well-founded, then there can be multiple solutions. The standard semantics of recursive programs gives a particular solution, namely the least solution in a flat Scott domain, which may not be the desired one. We have recently proposed programming language constructs to allow the specification of alternative solutions and methods to compute them. We have implemented these new constructs as an extension of OCaml.

In this paper, we prove some theoretical results characterizing well-founded coalgebras that slightly extend results of Adámek, Lücke, and Milius (2007), along with several examples for which this extension is useful. We also give several examples that are not well-founded but still have a desired solution. In each case, the function would diverge under the standard semantics of recursion, but can be specified and computed with the programming language constructs we have proposed.
\end{abstract}

\section{Introduction}

Infinite coinductive datatypes and functions on them offer interesting challenges in the design of programming languages. While most programmers feel comfortable with inductive datatypes, coinductive datatypes are often considered difficult to handle. Many programming languages do not even provide constructs to define them. OCaml offers the possibility of defining coinductive datatypes, but the means to define recursive functions on them are limited. Often the obvious definitions do not halt or provide the wrong solution.

Theoretical models of recursion schemes have been well studied under the names wellfounded coalgebras, recursive coalgebras (ALM07), corecursive algebras (CUV09), and Elgot algebras (AMV06). Much of this work focuses on conditions ensuring unique or canonical solutions, e.g. when the coalgebra is well-founded.

A prototypical example of a function that fits the well-founded scheme is mergesort. Given a list, we can sort it by dividing it into identical pieces, sorting the smaller lists, then 
merging the resulting sorted lists. The base case is the empty list or the list containing a single element. As with most recursive functions, the scheme of definition is: given an argument, check if it is the base case; if not, prepare the arguments for the recursive calls, recursively apply the function, then combine the results of the recursive calls into the final result. For mergesort, this scheme is illustrated in the following diagram:

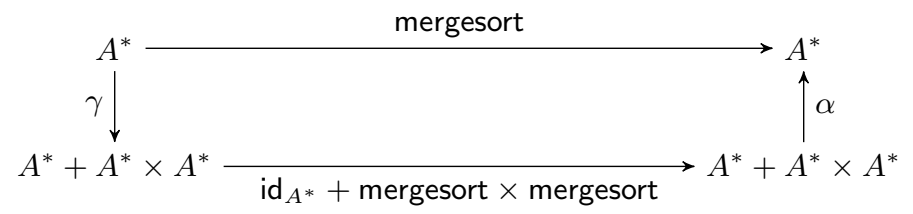

The function $\gamma$ checks whether the list is empty or a singleton, otherwise divides it in two lists of roughly equal size.

$$
\begin{aligned}
\gamma(\ell) & =\iota_{1}(\ell), \quad \ell=[] \text { or } \ell=[a] \\
\gamma\left(\left[a_{1} ; \ldots ; a_{n}\right]\right) & =\iota_{2}\left(\left[a_{1} ; \ldots ; a_{\lfloor n / 2]}\right],\left[a_{\lfloor n / 2\rfloor+1} ; \ldots ; a_{n}\right]\right), \quad n \geq 2 .
\end{aligned}
$$

Here $\iota_{1}$ and $\iota_{2}$ are the injections into the coproduct. After the function is applied recursively, the results of the recursive calls are combined by $\alpha$, which merges the two sorted lists.

$$
\alpha\left(\iota_{1}(\ell)\right)=\ell \quad \alpha\left(\iota_{2}\left(\ell_{1}, \ell_{2}\right)\right)=\operatorname{merge}\left(\ell_{1}, \ell_{2}\right)
$$

The merge function obeys a similar scheme:

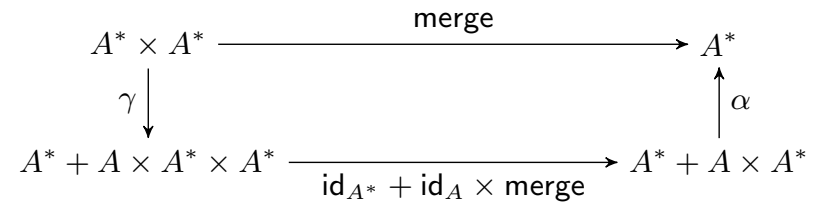

where

$$
\begin{array}{cc}
\gamma([], \ell)=\gamma(\ell,[])=\iota_{1}(\ell) & \alpha\left(\iota_{1}(\ell)\right)=\ell \\
\gamma\left(a_{1}:: \ell_{1}, a_{2}:: \ell_{2}\right)=\left\{\begin{array}{lll}
\iota_{2}\left(a_{1}, \ell_{1}, a_{2}:: \ell_{2}\right) & \text { if } a_{1} \leq a_{2} \\
\iota_{2}\left(a_{2}, a_{1}:: \ell_{1}, \ell_{2}\right) & \text { if } a_{1}>a_{2}
\end{array}\right. & \alpha\left(\iota_{2}(a, \ell)\right)=a:: \ell .
\end{array}
$$

The fact that these functions are well-defined and unique follows from the theory of recursive coalgebras (ALM07).

Abstractly, these definitional schemes are of the form

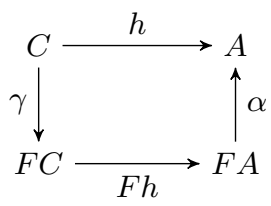

where $F$ is usually a polynomial functor on Set and $(C, \gamma)$ and $(A, \alpha)$ are a coalgebra and an algebra, respectively, for the functor $F$. The function $h$ being defined is called an F-coalgebra-algebra morphism. 
The standard semantics of recursion, as provided by all modern programming languages, provides a means of expressing and computing the unique solution of (1), provided the coalgebra $C$ is well-founded; that is, provided there is a basis to the recursion. However, the diagram (1) can act as a valid definitional scheme even when $C$ is not wellfounded. This observation was the starting point of our work on new program constructs for functions defined by such definitional schemes when $C$ is not well-founded (JKS12, JKS13). In the non-well-founded case, there can be multiple solutions. The standard semantics of recursive programs gives a particular solution, namely the least solution in a flat Scott domain, which in the non-well-founded case is typically not be the desired one. In (JKS12, JKS13), we introduced new programming language constructs to allow the programmer to specify and compute a desired alternative solution by solving a set of equations determined by (1).

In the course of our study, we also proved some theoretical results that clarify and slightly generalize some results of (ALM07). In this paper, we present those results, and provide some examples where our extension is useful. Although the results of (ALM07) apply to a large class of recursive function definitions, there appear to be cases that are not covered, at least not in any straightforward way. The simplest example is the case of mutually recursive definitions. For example, consider the even and odd predicates on natural numbers. In an ML-style language, we would write:

let rec even $\mathrm{n}=$ if $\mathrm{n}=0$ then true else odd $(\mathrm{n}-1)$

and odd $n=$ if $n=0$ then false else even ( $n-1)$

Our results extend the results of (ALM07) to several patterns of function definitions, including this one. Mutually recursive functions are treated in (AMV06), but our treatment is more symmetric.

The main result of this paper is a theoretical result that clarifies and mildly generalizes a result of (ALM07). We show:

- Every $F$-coalgebra $C$ contains a maximal well-founded subcoalgebra wf $C$.

- If $R$ is a final $F$-coalgebra, then wf $R$ is the initial $F$-algebra.

- Let $C$ be an $F$-coalgebra. The following are equivalent:

- $C$ is well-founded; that is, $C=\operatorname{wf} C$.

- There is a valid induction principle for $C$ (defined precisely in 3.2 . .

- There is a unique coalgebra morphism $C \rightarrow$ wf $R$.

- There is a unique coalgebra-algebra morphism from $C$ to any $F$-algebra.

Our constructions are based on the concept of realizations, a concrete representation of final coalgebras for a wide class of multisorted type signatures (Koz11). Realizations go beyond ordinary polynomial functors on Set in that they handle infinite (countable or uncountable) product and sum as well as total and partial functions. They also handle multi-sorted signatures in a more symmetric way, without relying on any Cartesian structure or parameterization as in (AMV06).

Our second contribution is a variety of well-founded and non-well-founded examples that illustrate the power and limitations of the theory.

The paper is organized as follows. In $\$ 2$ we review the results of (Koz11) on realizations of coinductive types, which are essential to the understanding of our main theoretical 
results in $\$ 3$ In $\$ 3$ we give a new characterization of well-founded coalgebras in terms of realizations. In $\$ 4$ we present several examples of well-founded applications. Some of these are already covered by the results of (ALM07), but others, such as mutually recursive functions even/odd and the Ackermann function, are not. However, each of these exhibits some interesting or surprising characteristic that attests to the wide applicability of the theory. In $\$ 5$ we present several non-well-founded examples, including an example of Capretta (Cap07) involving descending sequences of natural numbers and the semantics of alternating Turing machines and IND programs (HK84). These examples illustrate the usefulness of (1) as a definitional scheme even in the non-well-founded case. In $\$ 6$ we briefly describe our experience with bringing these theoretical ideas to practical fruition in the form of new programming language constructs for specifying alternative solutions to (11). These practical results are reported more fully in (JKS12,JKS13), but here we are able to put them in the proper theoretical context. We conclude in $\$ 7$ with a discussion of related theoretical and practical results.

\section{Realization of Coinductive Types}

In the proof of Theorem 3.3, we make use of an explicit construction of final coalgebras from (Koz11). To make this paper self-contained, this section recalls the main definitions and results.

\subsection{Directed Multigraphs}

A directed multigraph is a structure $G=(V, E$, src, tgt $)$ with nodes $V$, edges $E$, and two maps src, tgt : $E \rightarrow V$ giving the source and target of each edge, respectively. We write $e: s \rightarrow t$ if $s=\operatorname{src} e$ and $t=\operatorname{tgt} e$. When specifying multigraphs, we will sometimes use the notation $s \stackrel{n}{\rightarrow} t$ for the metastatement, "There are exactly $n$ edges from $s$ to $t . "$

A path is a finite alternating sequence of nodes and edges

$$
s_{0} e_{0} s_{1} e_{1} s_{2} \cdots s_{n-1} e_{n-1} s_{n}
$$

$n \geq 0$, such that $e_{i}: s_{i} \rightarrow s_{i+1}, 0 \leq i \leq n-1$. These are the arrows of the free category generated by $G$. The length of a path is the number of edges. A path of length 0 is just a single node. The first and last nodes of a path $p$ are denoted $\operatorname{src} p$ and $\operatorname{tgt} p$, respectively. As with edges, we write $p: s \rightarrow t$ if $s=\operatorname{src} p$ and $t=\operatorname{tgt} p$.

A multigraph homomorphism $\ell: G_{1} \rightarrow G_{2}$ is a map $\ell: V_{1} \rightarrow V_{2}, \ell: E_{1} \rightarrow E_{2}$ such that if $e: s \rightarrow t$ then $\ell(e): \ell(s) \rightarrow \ell(t)$. This lifts to a functor on the free categories generated by $G_{1}$ and $G_{2}$.

\subsection{Type Signatures}

A type signature is a directed multigraph $F$ along with a designation of each node of $F$ as either existential or universal. The existential and universal nodes correspond respectively to coproduct and product constructors. The directed edges of the graph represent the corresponding destructors. 


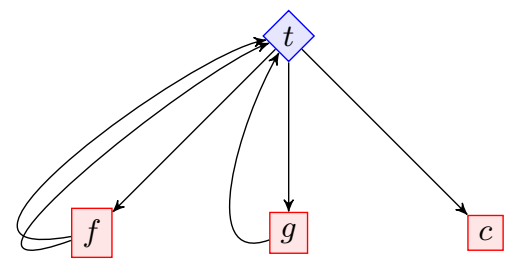

Fig. 1. A multigraph representing a single-sorted algebraic signature. Blue diamonds represent existential nodes and red squares universal nodes.

For example, consider an algebraic signature consisting of a binary function symbol $f$, a unary function symbol $g$, and a constant $c$. This would ordinarily be represented by the polynomial endofunctor $F X=X^{2}+X+\mathbb{1}$, or in OCaml by

type $t=F$ of $t * t \mid G$ of $t \mid C$

We would represent this signature by a directed multigraph consisting of four nodes $\{t, f, g, c\}$, of which $t$ is existential and $f, g, c$ are universal, along with edges

$$
t \stackrel{1}{\rightarrow} f \quad t \stackrel{1}{\rightarrow} g \quad t \stackrel{1}{\rightarrow} c \quad f \stackrel{2}{\rightarrow} t \quad g \stackrel{1}{\rightarrow} t .
$$

The multigraph is illustrated in Fig. 1]

\subsection{Coalgebras and Realizations}

Let $F$ be a type signature with nodes $V$. An $F$-coalgebra is a $V$-indexed collection of pairs $\left(C_{s}, \gamma_{s}\right)$, where the $C_{s}$ are sets and the $\gamma_{s}$ are set functions

$$
\gamma_{s}: C_{s} \rightarrow \begin{cases}\sum_{\mathrm{src} e=s} C_{\mathrm{tgt} e}, & \text { if } s \text { is existential, } \\ \prod_{\mathrm{src} e=s} C_{\mathrm{tgt} e}, & \text { if } s \text { is universal. }\end{cases}
$$

A morphism of $F$-coalgebras is a $V$-indexed collection of set maps $h_{s}$ that commute with the $\gamma_{s}$ in the usual way. Similarly, an $F$-algebra is a $V$-indexed collection of pairs $\left(A_{s}, \alpha_{s}\right)$, where the $A_{s}$ are sets and the $\alpha_{s}$ are set functions

$$
\alpha_{s}:\left\{\begin{array}{ll}
\sum_{\mathrm{src} e=s} A_{\mathrm{tgt} e}, & \text { if } s \text { is existential, } \\
\prod_{\mathrm{src} e=s} A_{\mathrm{tgt} e}, & \text { if } s \text { is universal }
\end{array}\right\} \rightarrow A_{s} .
$$

A morphism of $F$-algebras is a $V$-indexed collection of set maps $h_{s}$ that commute with the $\alpha_{s}$ in the usual way. These definitions correspond to the traditional definition of $F$-coalgebras and $F$-algebras for an endofunctor $F$ on $\mathrm{Set}^{V}$.

Coalgebras are equivalent to realizations. An $F$-realization is a directed multigraph $G$ along with a multigraph homomorphism $\ell: G \rightarrow F$, called a typing, with the following properties.

- If $\ell(u)$ is existential, then there is exactly one edge of $G$ with source $u$.

- If $\ell(u)$ is universal, then $\ell$ is a bijection between the edges of $G$ with source $u$ and the edges of $F$ with source $\ell(u)$.

A homomorphism of $F$-realizations is a multigraph homomorphism that commutes with the typings. 
Theorem 2.1 ( $(\overline{\text { Koz11 }}))$. The categories of $F$-coalgebras and $F$-realizations are equivalent (in the sense of (ML71, §IV.4)).

\subsection{Final Coalgebras}

Realizations allow a concrete construction of final coalgebras that is reminiscent of the Brzozowski derivative on sets of strings. Here, instead of strings, the derivative acts on certain sets of paths of the type signature.

Let $F$ be a type signature. Construct a realization $R, \ell$ as follows. A node of $R$ is a set $A$ of finite paths in $F$ such that

- $A$ is nonempty and prefix-closed;

- all paths in $A$ have the same first node, which we define to be $\ell(A)$;

- if $p$ is a path in $A$ of length $n$ and $\operatorname{tgt} p$ is existential, then there is exactly one path of length $n+1$ in $A$ extending $p$;

- if $p$ is a path in $A$ of length $n$ and tgt $p$ is universal, then all paths of length $n+1$ extending $p$ are in $A$.

The edges of $R$ are defined as follows. Let $A$ be a set of paths in $F$ and $e$ an edge of $F$. Define the Brzozowski derivative of $A$ with respect to $e$ to be

$$
D_{e}(A)=\{p \mid(\operatorname{src} e) e p \in A\},
$$

the set of paths obtained by removing the initial edge $e$ from paths in $A$ that start with that edge. If $A$ is a node of $R$ and $D_{e}(A)$ is nonempty, we include exactly one edge

$$
\langle A, e\rangle: A \rightarrow D_{e}(A)
$$

in $R$ and take $\ell(\langle A, e\rangle)=e$. It is readily verified that $\operatorname{tgt}\langle A, e\rangle=D_{e}(A)$ satisfies properties (i)-(iv) and that $\ell\left(D_{e}(A)\right)=\operatorname{tgt} e$, so $\ell$ is a typing.

Theorem $2.2((\overline{\text { Koz11 }}))$. The realization $R, \ell$ is final in the category of $F$-realizations. The corresponding $F$-coalgebra as constructed in Theorem 2.1 is final in the category of $F$-coalgebras.

\section{Characterization of Well-Founded Coalgebras}

Well-foundedness of coalgebras has a precise characterization in terms of their corresponding realizations: a coalgebra is well-founded if and only if its corresponding realization is well-founded as a graph; that is, if it has no infinite directed paths. The main theorem of (ALM07) characterizes halting in terms of finiteness instead of wellfoundedness, which by König's lemma is equivalent for the finitary functors considered in (ALM07), but it is really well-foundedness and not finiteness that is the essential property. In the following, we consider coalgebras for a wider class of functors, namely multi-sorted polynomial functors on $\operatorname{Set}^{V}$, where $V$ is a set of sorts, with infinite (countable and uncountable) product and sum, as well as total and partial functions. This is the same class of functors considered in (Koz11). Let $F$ be such a functor.

When a recursive function is called on a well-founded argument, the solution is unique 
and the standard semantics will terminate. Theorem 3.3, which generalizes (ALM07) to the non-finitary case, characterizes the conditions under which this occurs.

The proof of Theorem 3.3 relies on some extra interesting facts which we also prove, namely that every $F$-coalgebra $C$ contains a unique maximal well-founded subcoalgebra wf $C$ and that if $R$ is the final $F$-coalgebra, then wf $R$ is the initial $F$-algebra.

\subsection{Well-Founded Coalgebras}

An $F$-coalgebra-algebra morphism is a set function $h: C \rightarrow A$, where $(C, \gamma)$ is an $F$ coalgebra and $(A, \alpha)$ is an $F$-algebra, such that the diagram

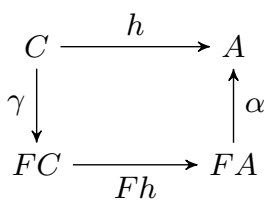

commutes.

An $F$-realization $G=(V, E$, src, tgt, $\ell)$ is well-founded if all directed $E$-paths are finite. An $F$-coalgebra is well-founded if its corresponding $F$-realization is.

Lemma 3.1. Every F-coalgebra contains a unique maximal well-founded subcoalgebra.

Proof. Equivalently, every $F$-realization $G=(V, E$, src, tgt, $\ell)$ contains a unique maximal well-founded $F$-subrealization wf $G$. The nodes wf $V$ are the nodes of $G$ from which there are no infinite directed $E$-paths. The graph wf $G$ is the induced subgraph on wf $V$. Equivalently, the set of nodes of wf $G$ is the smallest set of nodes $A$ of $G$ satisfying the closure condition: if all $E$-successors of $s$ are in $A$, then $s \in A$.

Lemma 3.2. Let $R=(V, E$, src, tgt, $\ell)$ be the final $F$-realization. Then wf $R$ is an $F$-algebra.

Proof. By Lambek's lemma (Lam68), the structure map $\left(\gamma_{s} \mid s \in V\right)$ of the final $F$ coalgebra corresponding to $R$ is invertible, thus forms an $F$-algebra. Translating back to the realization $R$, this means that

- for every edge $e \in E$ such that $\operatorname{src} e$ is existential and every node $v$ of $R$ with $\ell(v)=$ tgt $e$, there exists a unique node $u$ and edge $d$ of $R$ such that src $d=u$, tgt $d=v$, and $\ell(d)=e ;$ and

- for every universal node $s \in V$ and tuple $\left(v_{e} \mid \operatorname{src} e=s\right)$ of nodes of $R$ such that $\ell\left(v_{e}\right)=\operatorname{tgt} e$, there exist a unique node $u$ and tuple of edges $\left(d_{e} \mid \operatorname{src} e=s\right)$ of $R$ such that $\operatorname{src} d_{e}=u, \operatorname{tgt} d_{e}=v_{e}$, and $\ell\left(d_{e}\right)=e$.

The existence and uniqueness of $u$ in the above two cases assert the closure of $R$ under the algebraic operations. The subrealization $\mathrm{wf} R$ is closed under these operations, because any node all of whose immediate $E$-successors are in wf $R$ is also in wf $R$, therefore wf $R$ is a subalgebra of $R$. 
We will show in Corollary 3.4 that wf $R$ is in fact the initial $F$-algebra (up to isomorphism). To show initiality, we need to show that there is a unique $F$-algebra morphism to any other $F$-algebra. This will follow as a special case of Theorem 3.3 (iv) below.

\subsection{Induction Principle}

The well-founded part of a realization $G$ can be expressed in the modal $\mu$-calculus as wf $G=\mu X$. $\square X$, where the modality $\square$ is interpreted in $G$ by the $E$-successor relation $E(x)=\{\operatorname{tgt} e \mid e \in E, \operatorname{src} e=x\}$; that is, the modal formula $\square P$ holds of $x$ if $P$ holds of all $E$-successors of $x$. Thus $G$ is well-founded if $\mu X$. $\square X$ is universally valid in $G$.

The induction principle for a well-founded realization $G=(V, E$, src, tgt, $\ell)$ is:

$$
\frac{\forall x(\forall y \in E(x) P(y)) \rightarrow P(x)}{\forall x P(x)},
$$

or more concisely,

$$
\frac{\square P \rightarrow P}{P} .
$$

As we argue in Theorem 3.3 , this rule is sound if and only if $G$ is well-founded.

\subsection{Main Theorem}

We are now ready to state and prove our main theorem. We include point (v) to align with (ALM07, Theorem 3.8), although it is not really needed for our work.

Theorem 3.3. Let $(C, \gamma)$ be an $F$-coalgebra and let $R$ be the final $F$-coalgebra. The following are equivalent:

(i) $C$ is well-founded; that is, $C=\operatorname{wf} C$.

(ii) The induction principle $(3)$ is valid for $C$.

(iii) There is a unique coalgebra morphism $C \rightarrow$ wf $R$.

(iv) There is a unique coalgebra-algebra morphism from $C$ to any $F$-algebra.

(v) There is a unique parameterized coalgebra-algebra morphism from $C$ to any $F$ algebra.

Proof. The equivalence of (i) and (ii) is a fundamental property of relational algebra. The implication (i) $\Rightarrow$ (ii) requires the axiom of dependent choice.

Assuming (i) and (ii), (iv) can be proved by defining a coalgebra-algebra morphism by induction, using (3). Let $\left(A_{s}, \alpha_{s}\right)$ be an arbitrary $F$-algebra. Assume the coalgebra $C$ is given in the form of an $F$-realization $G=(V, E$, src, tgt, $\ell)$. We must define maps $h_{s}: \ell^{-1}(s) \rightarrow A_{s}$ for $s \in V$ satisfying condition (2). This is equivalent to the following two conditions. Let $s \in V$ and $u \in V$ such that $\ell(u)=s$.

- If $s$ is existential, let $d$ be the unique edge with $\operatorname{src} d=u$, let $v=\operatorname{tgt} d$, and let $e=\ell(d)$. Then

$$
h_{s}(u)=\alpha_{s}\left(\operatorname{in}_{e}\left(h_{\operatorname{tgt} e}(v)\right)\right) \in A_{s} .
$$


- If $s$ is universal, for each $e$ such that $\operatorname{src} e=s$, let $d_{e}$ be the unique edge with $u=\operatorname{src} e$ and $\ell\left(d_{e}\right)=e$, and let $v_{e}=\operatorname{tgt} d_{e}$. Then

$$
h_{s}(u)=\alpha_{s}\left(h_{\operatorname{tgt} e}\left(v_{e}\right) \mid \operatorname{src} e=s\right) \in A_{s} .
$$

The maps $h_{s}$ are uniquely defined by these equations due to the well-foundedness of the $E$-successor relation on $G$.

By Lemma 3.2 wf $R$ is an $F$-algebra, thus (iii) follows as a special case of (iv).

To argue that (iii) implies (i), we observe that under any morphism of $F$-realizations $C \rightarrow$ wf $R$, an infinite path in $C$ would map to an infinite path in wf $R$, which cannot exist by definition, since wf $R$ is well-founded. Thus $C$ must be well-founded as well.

For $(\mathrm{v}) \Rightarrow(\mathrm{iv})$, suppose that there is a unique parameterized coalgebra-algebra morphism from $C$ to any $F$-algebra. That is, for any $\alpha^{\prime}: F A \times C \rightarrow A$ there is a unique $h$ which makes the following diagram commute:

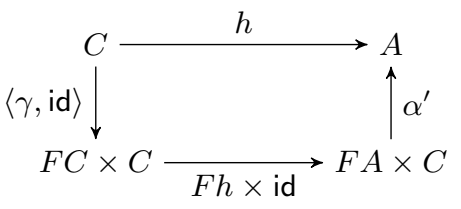

We want to show that that there is a unique coalgebra-algebra morphism from $C$ to any $F$-algebra.

Take an arbitrary $F$-algebra $\alpha: A \rightarrow F A$ and consider $\alpha^{\prime}=\alpha \circ \pi_{1}: F A \times C \rightarrow A$. Using the diagram (4), we know that there exists a unique $h: C \rightarrow A$ such that $h=$ $\alpha \circ \pi_{1} \circ(F h \times$ id $) \circ\langle\gamma$, id $\rangle$. We show that $h$ is a coalgebra-algebra morphism from $C$ to $A$ and that it is unique.

$$
\begin{aligned}
h & =\alpha \circ \pi_{1} \circ(F h \times \mathrm{id}) \circ\langle\gamma, \mathrm{id}\rangle & & \text { diagram (4) } \\
& =\alpha \circ F h \circ \pi_{1} \circ\langle\gamma, \mathrm{id}\rangle & & \pi_{1} \text { is a natural transformation } \\
& =\alpha \circ F h \circ \gamma & & \pi_{1} \circ\langle f, g\rangle=f .
\end{aligned}
$$

For uniqueness, note that any other coalgebra-algebra morphism $g: C \rightarrow A$ also makes diagram (4) commute, for $\alpha^{\prime}=\alpha \circ \pi_{1}$ :

$$
\begin{aligned}
g & =\alpha \circ F g \circ \gamma & & \text { definition of coalgebra-algebra morphism } \\
& =\alpha \circ F g \circ \pi_{1} \circ\langle\gamma, \mathrm{id}\rangle & & \pi_{1} \circ\langle f, g\rangle=f \\
& =\alpha \circ \pi_{1} \circ(F g \times \mathrm{id}) \circ \gamma & & \pi_{1} \text { is a natural transformation. }
\end{aligned}
$$

Hence $g=h$.

For (iv) $\Rightarrow(\mathrm{v}$ ), we need the following fact. Let $\gamma: C \rightarrow F C$ be an $F$-coalgebra. Define $G(X)=C \times F X$. If $(C, \gamma)$ is a well-founded $F$-coalgebra, then $(C,\langle\gamma$, id $\rangle)$ is a wellfounded $G$-coalgebra. If (i) holds for $F$, then it also holds for $G$, therefore (iv) holds for $G$, and (v) follows trivially for $F$ since the diagram (4) for $F$ is a coalgebra-algebra morphism diagram for $G$.

Corollary 3.4. The $F$-coalgebra wf $R$ is (up to isomorphism) the initial $F$-algebra. 
Proof. The structure wf $R$ is an $F$-algebra by Lemma 3.2. But it is also a well-founded $F$-coalgebra by definition. By the equivalence of Theorem 3.3(i) and (iv), there is a unique $F$-algebra morphism from wf $R$ to any $F$-algebra, thus wf $R$ is initial.

\subsection{Non-Well-Founded Coalgebras}

In many interesting non-well-founded cases, $h$ is not unique and depends on the choice of solution method in the codomain $A$. However, for a large class of ordered codomains, one is interested in a canonical solution, namely the least fixpoint of a monotone map specified by the function definition. This situation was studied in (AMV06), in which it was shown that under certain conditions on the codomain, a function defined on a non-well-founded coalgebra can be considered a function on the final coalgebra and is independent of the input representation. This covers many examples in which the intended solution is a least fixpoint. The following result is a minor adaptation of (AMV06, Proposition 3.5) to our framework and the proof is similar.

Theorem 3.5. Let $(A, \alpha)$ be an ordered $F$-algebra such that $A$ is a chain-complete and $\alpha$ order-continuous. The construction of the least fixpoint of the map $h \mapsto \alpha \circ F h \circ \gamma$ is natural in $S$; that is, if $f: S \rightarrow S^{\prime}$ is an F-coalgebra morphism, then $h_{S}=h_{S^{\prime}} \circ f$.

Although Theorem 3.5 covers many interesting non-well-founded situations, there are some that it does not cover. For instance, to define substitution on infinitary $\lambda$-terms, the codomain is a coalgebra of infinitary terms, which is not ordered in any natural way. In this case, the solution is unique for other reasons.

\section{Well-Founded Examples}

In this section, we present examples of recursive functions which are well-founded. The first two, the greatest common divisor of two integers and the towers of Hanoi, already fit the framework of (ALM07). The other are guaranteed to have a unique solution using the multi-sorted extension to their framework that we have proposed.

\subsection{Integer $G C D$}

For integers $m, n \geq 0$ but not both 0 , we would like to compute a triple $(g, s, t)$ such that $g$ is the greatest common divisor $(\mathrm{gcd})$ of $m$ and $n$ and $s m+t n=g$. A recursive definition is

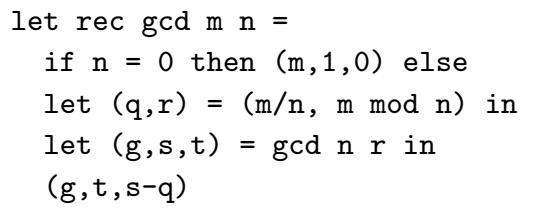

This gives the following instantiation of (2): 


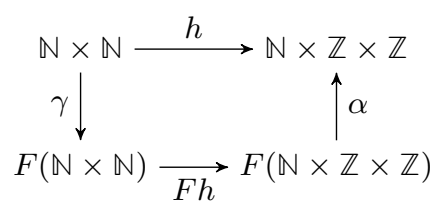

Here $F X=\mathbb{N}+X \times \mathbb{N}$ and

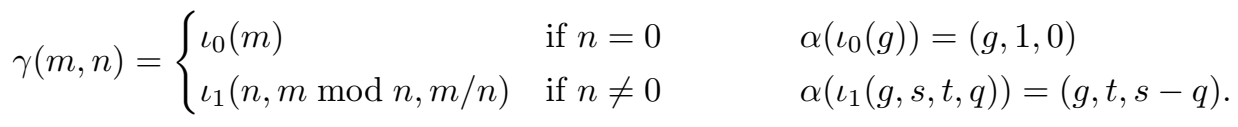

The theory of recursive coalgebras (ALM07) guarantees the existence of a unique function satisfying the diagram.

\subsection{Towers of Hanoi}

Another classic example of a recursive function is the towers of Hanoi. This mathematical game consists of three rods $A, B$ and $C$ and a number of disks of different sizes that can slide on any rod. At the beginning of the game, all disks are on $\operatorname{rod} A$ in order of size, smallest on top. The goal of the game is to find a procedure to move all disks to rod $B$ while respecting the following rules:

- only one disk at a time can be moved

- a move consists of removing the upper disk from one of the rods and sliding in onto another rod, on top of other disks that might already be on that rod;

- no disk may be placed on top of a smaller disk.

For $n$ disks, a recursive solution consists in recursively moving $n-1$ disks from the origin $\operatorname{rod} A$ to the third $\operatorname{rod} C$, then moving the biggest disk from the origin $\operatorname{rod} A$ to the destination $\operatorname{rod} B$, and finally recursively moving $n-1$ disks from the third $\operatorname{rod} C$ to the destination $\operatorname{rod} B$. It is given by the following OCaml implementation, where $o, d$ and $t$ are the origin, destination and third rod, respectively:

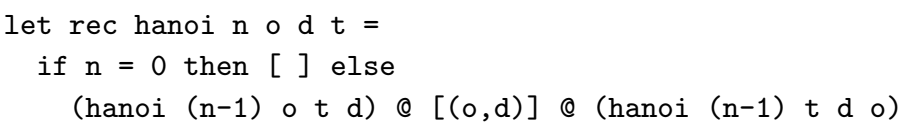

Let $R$ be the set of rods $\{A, B, C\}$. A move can be represented as an element of $R^{2}$ consisting of the origin and the destination of the move. This gives the following instantiation of (2):

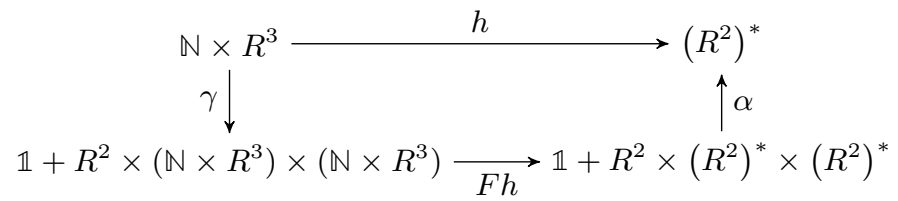

Here $F X=\mathbb{1}+R^{2} \times X$ and

$$
\begin{aligned}
\gamma(n, o, d, t) & = \begin{cases}\iota_{0}(), & \text { if } n=0, \\
\iota_{1}(o, d,(n-1, o, t, d),(n-1, t, d, o)), & \text { if } n \neq 0\end{cases} \\
\alpha\left(\iota_{0}()\right) & =\varepsilon \quad \alpha\left(\iota_{1}(o, d, b, e)\right)=b \cdot(o, d) \cdot e .
\end{aligned}
$$


The theory of recursive coalgebras (ALM07) guarantees the existence of a unique function satisfying the diagram.

\subsection{Mutually Recursive Functions: even-odd}

This subsection illustrates how our generalization to multi-sorted signatures handles mutually recursive functions in a symmetric way. A very simple example is the definition of the even and odd predicates on natural numbers.

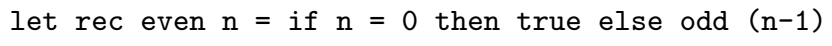

We can depict the recursion graphically with the following diagram:

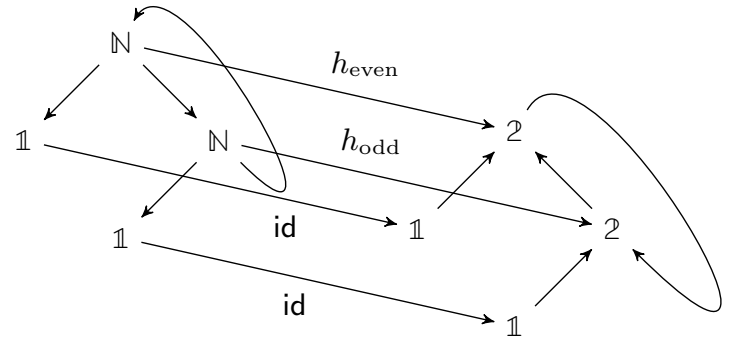

This can be viewed as an endofunctor $F:$ Set $^{V} \rightarrow \operatorname{Set}^{V}$, where $V=\{$ even, odd $\}$. The functor is defined by: $F(A, B)=(\mathbb{1}+B, \mathbb{1}+A)$ and if $g: A \rightarrow A^{\prime}$ and $h: B \rightarrow B^{\prime}$, then $F(g, h)=(\mathrm{id}+h, \mathrm{id}+g): F(A, B) \rightarrow F\left(A^{\prime}, B^{\prime}\right)$.

An $F$-coalgebra is a pair $((C, D), \gamma)$, where $\gamma:(C, D) \rightarrow F(C, D)$ is a morphism in the underlying category $\operatorname{Set}^{V}$; that is,

$$
\gamma=\left(\gamma_{\text {even }}, \gamma_{\text {odd }}\right):(C, D) \rightarrow(\mathbb{1}+D, \mathbb{1}+C),
$$

where $\gamma_{\text {even }}: C \rightarrow \mathbb{1}+D$ and $\gamma_{\text {odd }}: D \rightarrow \mathbb{1}+C$. Similarly, an $F$-algebra is a pair $((A, B), \alpha)$, where $\alpha: F(A, B) \rightarrow(A, B)$ is a morphism in $\operatorname{Set}^{V}$; that is,

$$
\alpha=\left(\alpha_{\text {even }}, \alpha_{\text {odd }}\right):(\mathbb{1}+B, \mathbb{1}+A) \rightarrow(A, B),
$$

where $\alpha_{\text {even }}: \mathbb{1}+B \rightarrow A$ and $\alpha_{\text {odd }}: \mathbb{1}+A \rightarrow B$.

An $F$-algebra-coalgebra morphism $h:((C, D), \gamma) \rightarrow((A, B), \alpha)$ is a map $h=\left(h_{\text {even }}, h_{\text {odd }}\right)$ : $(C, D) \rightarrow(A, B)$ such that the following diagram commutes:

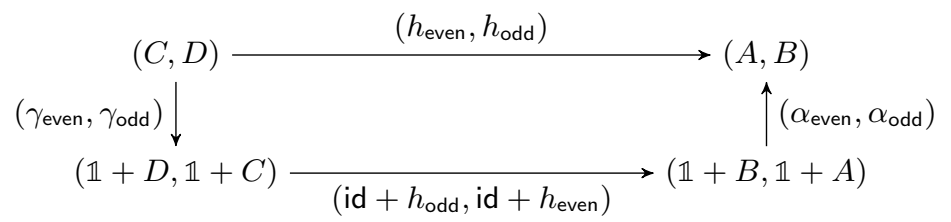

In our application, we have $A=B=2$ and $C=D=\mathbb{N}$, with

$$
\begin{array}{r}
\gamma_{\text {even }}(n)=\gamma_{\text {odd }}(n)= \begin{cases}\iota_{0}() & \text { if } n=0 \\
\iota_{1}(n-1) & \text { if } n>0\end{cases} \\
\alpha_{\text {even }}\left(\iota_{0}()\right)=\mathbf{1} \quad \alpha_{\text {odd }}\left(\iota_{0}()\right)=\mathbf{0} \quad \alpha_{\text {even }}\left(\iota_{1}(b)\right)=\alpha_{\text {odd }}\left(\iota_{1}(b)\right)=b .
\end{array}
$$




\subsection{Ackermann Function}

The Ackermann function

$$
A(0, n)=n+1 \quad A(m+1,0)=A(m, 1) \quad A(m+1, n+1)=A(m, A(m+1, n))
$$

is a notoriously fast-growing function that also fits into our general scheme (although one should not try to compute it!). This example is quite interesting, because at first glance it seems not to fit into the general scheme (2) because of the nested recursive call in the third clause. However, a key insight comes from the termination proof, which is done by induction on the well-founded lexicographic order on $\mathbb{N} \times \mathbb{N}$ with $m$ as the more significant parameter. We see that we can break the definition into two stages, both higher-order.

Rewriting $A(m, n)$ as $A_{m}(n)$, we have that (5) is equivalent to

$$
A_{0}=\lambda n . n+1 \quad A_{m+1}=\lambda n \cdot A_{m}^{n+1}(1),
$$

where $f^{n}$ denotes the $n$-fold composition of $f$ with itself:

$$
f^{0}=\lambda n . n \quad f^{n+1}=f \circ f^{n} .
$$

The outermost stage computes $m \mapsto A_{m}$. The diagram is

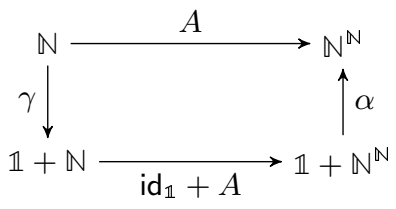

where

$$
\gamma(0)=\iota_{0}() \quad \gamma(m+1)=\iota_{1}(m) \quad \alpha\left(\iota_{0}()\right)=\lambda n . n+1 \quad \alpha\left(\iota_{1}(f)\right)=\lambda n . f^{n+1}(1) .
$$

In turn, the function $\alpha$ is defined in terms the $n$-fold composition function $(n, f) \mapsto f^{n}$ :

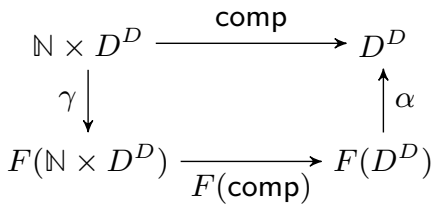

where $F X=\mathbb{1}+D^{D} \times X$ and

$$
\gamma(0, f)=\iota_{0}() \quad \gamma(n+1, f)=\iota_{1}(f, n, f) \quad \alpha\left(\iota_{0}()\right)=\mathrm{id}_{D} \quad \alpha\left(\iota_{1}(f, g)\right)=f \circ g .
$$

\section{Non-Well-Founded Examples}

We provided many examples of non-well-founded functions in (JKS13, JKS12), including probabilistic protocols, $p$-adic numbers, and a fairly substantial example involving abstract interpretation. Here we present a few more. We will also present an example involving the set of elements in an infinite list in $\$ 6$. 


\subsection{Descending Sequences}

As the simplest nontrivial coinductive datatype, streams offer the ideal playground to test new theories. We present an example on streams of natural numbers $\mathbb{N}^{\omega}$. The following example, taken from a talk by Capretta (Cap07), has a unique solution, but does not fit the existing theory of well-founded coalgebras (ALM07) or our generalization presented here, nor does it fit the theory of core corecursive algebras (CUV09).

The goal is to produce from a given stream of natural numbers another stream of natural numbers containing the lengths of the maximal strictly descending subsequences of the input stream. An example is shown in the following figure, where the input stream is depicted in a grid to easily picture the order of elements.

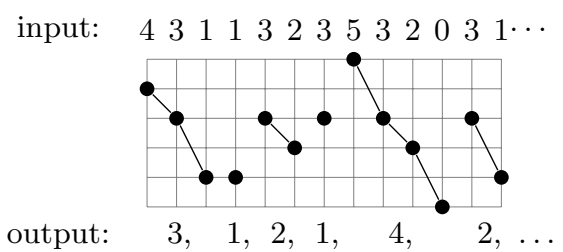

Here is a simple recursive definition of the function in CoCaml (see $\$ 6$ ), where the constructor solver builds a new stream:

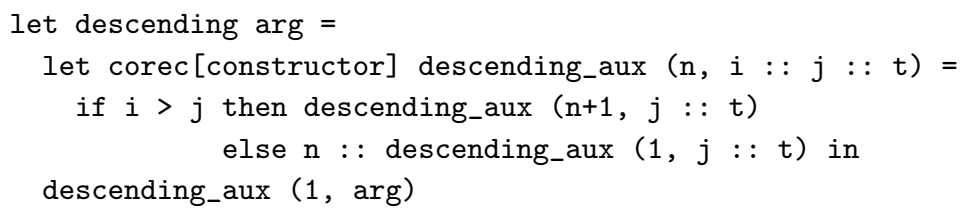

This definition corresponds to the following instantiation of (2):

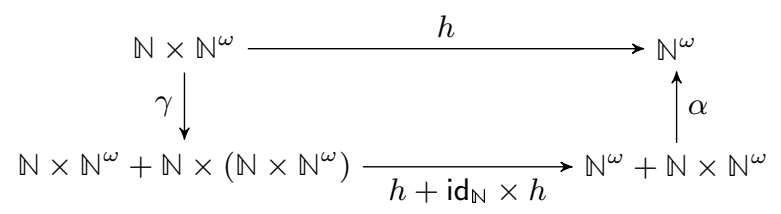

where $F X=X+\mathbb{N} \times X$ and

$$
\gamma(n, i:: j:: t)=\left\{\begin{array}{ll}
\iota_{0}(n+1, j:: t) & \text { if } i>j \\
\iota_{1}(n,(1, j:: t)) & \text { otherwise }
\end{array} \quad \alpha\left(\iota_{0}(s)\right)=s \quad \alpha\left(\iota_{1}(n, s)\right)=n:: s .\right.
$$

\subsection{Alternating Turing Machines and IND Programs}

The semantics of alternating Turing machines is described in terms of an inductive labeling of machine configurations $C$ with either $\mathbf{0}$ (rejecting), $\mathbf{1}$ (accepting), or $\perp$ (undetermined). In the present framework, the function $\gamma$ would give the set of successor configurations and the labeling of the state as either existential or universal, and $\alpha$ would tell how to label configurations $\mathbf{0}, \mathbf{1}$, or $\perp$ inductively up the computation tree. Formally, $\alpha$ gives the infimum for universal configurations and supremum for existential configurations in 3 -valued Kleene logic $B=\{\mathbf{0}, \perp, \mathbf{1}\}$ with ordering $\mathbf{0} \leq \perp \leq \mathbf{1}$. 


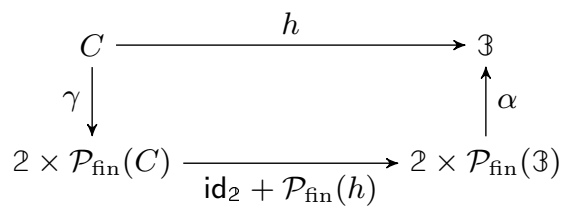

The canonical solution is defined to be the least fixpoint with respect to a different order, namely the flat Scott order $\perp \sqsubseteq \mathbf{0}, \perp \sqsubseteq \mathbf{1}$. This example is interesting, because it is a case in which $\alpha$ is not strict; for example, a universal configuration can be labeled $\mathbf{0}$ as soon as one of its successors is known to be labeled $\mathbf{0}$, regardless of the labels of the other successors.

A similar model is the IND programming language for the inductive sets (HK84). An IND program consists of a sequence of labeled statements of three kinds: universal and existential assignment $(x:=\forall$ and $x:=\exists$, respectively), conditional test (if $s=$ $t$ then $\ell_{1}$ else $\ell_{2}$ ), and halting (accept, reject). IND programs accept exactly the inductively definable sets, which over $\mathbb{N}$ are exactly the $\Pi_{1}^{1}$ sets. The semantics is identical to alternating Turing machines, except that the branching degree is equal to the cardinality of the domain of computation, thus the finite powerset functor must be replaced by the unrestricted powerset functor.

\section{CoCaml}

Along with our study characterizing the existence and uniqueness of solutions of diagram 2, we also became interested in situations in which solutions exist but are not unique. There are many interesting such cases, as our non-well-founded examples have shown. Often there is a desired solution to (2), but it is not the one computed by the standard semantics of recursion. We wanted to provide language constructs for the programmer to specify alternative solutions in those cases. This led to the design of an extension of OCaml called CoCaml (JKS12, JKS13, CoC12). The language is described in more detail there, but we would like to give a flavor of it in this section.

We provide some motivation using a function over the simplest coinductive datatype: infinite lists. In OCaml, the type of finite and infinite integer lists is built in as a sum type. The empty list is written [ ], and the list with head $\mathrm{h}$ and tail $\mathrm{t}$ is written $\mathrm{h}: \mathrm{:} \mathrm{t}$.

Infinite lists can now be defined coinductively using the let rec construct:

let rec ones $=1:$ : ones

let rec alt = $1:: 2::$ alt

The first example defines the infinite sequence of ones $1,1,1,1, \ldots$ and the second the sequence $1,2,1,2, \ldots$.

The let rec construct allows us to build only regular lists, that is, those that are ultimately periodic. Such lists always have a finite representation in memory. The coinductive elements we consider are always regular; that is, they have a finite but possibly cyclic representation. This is different from a setting in which infinite elements are represented lazily and are computed on the fly.

Although the let rec construct allows us to specify (finite representations of) infinite structures, further investigation reveals a major shortcoming. For example, suppose we 
wanted to define a function that, given an infinite list, returns the set of its elements. For the lists ones and alt, the function should return the sets $\{1\}$ and $\{1,2\}$, respectively. One would like to write a function definition using the obvious equations which patternmatch on the two constructors of the list datatype:

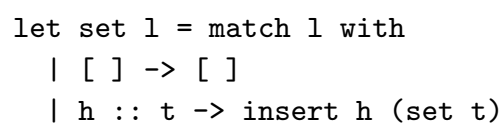

where insert inserts an element in a set, represented say by a sorted finite list without duplicates. However, this function will not halt in OCaml on the lists ones and alt, even though it is clear what the answers should be. Note that this is not a corecursive definition, as we are not asking for a greatest solution or a unique solution in a final coalgebra, but rather a least solution in a different ordered domain from the one provided by the standard semantics of recursive functions. The standard semantics of recursive functions gives us the least solution in the flat Scott domain with bottom element $\perp$ representing nontermination, whereas we would like the least solution in a different CPO, namely $(\mathcal{P}(\mathbb{Z}), \subseteq)$ with bottom element $\varnothing$.

The CoCaml language extends OCaml with a construct that allows functions defined by equations, like the one above, to be supplied with an extra parameter, namely a solver for the given equations. For instance, the example above would be almost the same in CoCaml:

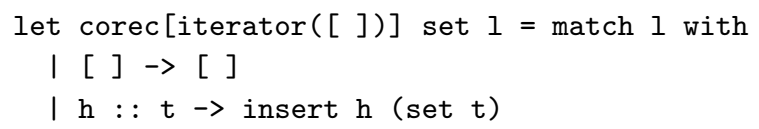

The construct corec with the parameter iterator([ ] ) specifies to the compiler that the equations above should be solved using the built-in iterator solver-in this case a least fixpoint computation - starting with the initial element [ ]. For the infinite list alt, which can abstractly be thought of as the circular structure

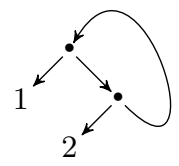

the compiler will generate two equations:

$\operatorname{set}(x)=$ insert $1(\operatorname{set}(y))$

$\operatorname{set}(y)=\operatorname{insert} 2(\operatorname{set}(x))$

then solve them using the specified solver iterator, which will produce the intended set $\{1,2\}$.

CoCaml has a number of built-in solvers (iterator, constructor, and a Gaussian elimination solver gaussian), as well as an interface for programmers to create their own solvers; see (JKS12, JKS13) and the CoCaml project website $(\overline{\mathrm{CoC} 12})$ for details.

\section{Discussion}

In this paper, we have presented the origins of our work on bringing coinduction to a functional language in the form of effective language constructs. 
The work in the present paper and related implementation papers (JKS13; JKS12) was inspired by work on recursive coalgebras (ALM07) and Elgot algebras (AMV06). We have extended and clarified the results in (ALM07) by providing a different proof that works on a larger class of functors. Our generalization handles multi-sorted signatures and mutually recursive functions in a symmetric way and is not restricted to finitary functors. We have also provided several examples of functions defined using this scheme, as well as non-well-founded examples that do not have a unique solution but still have a canonical solution. Finally, we have briefly described our work on programming language constructs to allow the programmer to choose alternative solution methods when the standard semantics of recursion would not halt.

The theoretical results of Adámek, Lücke, and Milius (ALM07) and the results of this paper are concerned with the properties of the domain $C$ ensuring unique solutions to the diagram (2). Capretta, Uustalu and Vene (CUV09) studied the dual problem of characterizing properties of the codomain $A$ ensuring this property. The work of Adámek, Milius, and Velebil on Elgot algebras (AMV06) is relevant to our work on recursive definitions that do not have unique solutions. Elgot algebras provide specified canonical solutions rather than unique ones. The canonical solutions must satisfy two axioms, the first ensuring that solutions are independent of the representation of the input and are thus well-defined on a final coalgebra, and the second that allows multiple fixpoints to be parameterized and computed sequentially. The latter property gives an alternative approach to mutually recursive functions. There is also some related work of a more practical nature (HLW03; ST98; Sym06; yW11) that we discuss in (JKS12, JKS13).

\section{Acknowledgments}

Thanks to Stefan Milius for stimulating discussions.

\section{References}

Jiří Adámek, Dominik Lücke, and Stefan Milius. Recursive coalgebras of finitary functors. Theoretical Informatics and Applications, 41:447-462, 2007.

Jiří Adámek, Stefan Milius, and Jiří Velebil. Elgot algebras. Log. Methods Comput. Sci., 2(5:4):131, 2006.

Venanzio Capretta. An introduction to corecursive algebras. http://www.cs.ru.nl/ venanzio/ publications/brouwer_seminar_4_12_2007.pdf, 2007.

CoCaml project. http://www.cs.cornell.edu/Projects/CoCaml/, December 2012.

Venanzio Capretta, Tarmo Uustalu, and Varmo Vene. Corecursive algebras: A study of general structured corecursion. In Marcel Vinicius Medeiros Oliveira and Jim Woodcock, editors, Formal Methods: Foundations and Applications, 12th Brazilian Symp. Formal Methods (SBMF 2009), volume 5902 of Lecture Notes in Computer Science, pages 84-100, Berlin, 2009. Springer.

David Harel and Dexter Kozen. A programming language for the inductive sets, and applications. Information and Control, 63(1/2):118-139, 1984.

Tom Hirschowitz, Xavier Leroy, and J. B. Wells. Compilation of extended recursion in call-byvalue functional languages. In PPDP 2003, pages 160-171, 2003. 
Jean-Baptiste Jeannin, Dexter Kozen, and Alexandra Silva. CoCaml: Programming with coinductive types. Technical Report http://hdl.handle.net/1813/30798, Computing and Information Science, Cornell University, December 2012.

Jean-Baptiste Jeannin, Dexter Kozen, and Alexandra Silva. Language constructs for non-wellfounded computation. In Matthias Felleisen and Philippa Gardner, editors, ESOP, volume 7792 of Lecture Notes in Computer Science, pages 61-80. Springer, 2013.

Dexter Kozen. Realization of coinductive types. Electr. Notes Theor. Comput. Sci., 276:237-246, 2011.

Joachim Lambek. A fixpoint theorem for complete categories. Mathematische Zeitschrift, 103:151-161, 1968.

Saunders Mac Lane. Categories for the Working Matematician. Springer, 1971.

Michael Sperber and Peter Thiemann. ML and the address operator. In 1998 ACM SIGPLAN Workshop on $M L$, September 1998.

Don Syme. Initializing mutually referential abstract objects: The value recursion challenge. In Proc. ACM-SIGPLAN Workshop on ML (2005). Elsevier, March 2006.

Baltasar Trancón y Widemann. Coalgebraic semantics of recursion on circular data structures. In Corina Cirstea, Monika Seisenberger, and Toby Wilkinson, editors, CALCO Young Researchers Workshop (CALCO-jnr 2011), pages 28-42, August 2011. 